\title{
A Review of Mobility Management in Integrated UMTS and WLAN Networks
}

\author{
Maushumi Barooah, Diganta Kumar Pathak, and Ishita Roy Karmakar
}

\begin{abstract}
In today's age of Next Generation Internet there have been a plethora of diversified overlapping networks where each of these communication technologies are optimized to provide user specific services and Quality of Service(QoS) parameters. The main objective of next generation networks focus at high bandwidth and high mobility. There are WiFi hotspots (WLAN) providing high data rates within smaller areas and UMTS networks providing larger coverage areas with low data rates. These two complementary networks can be combined to quench the thirst for ubiquitous communication enabling the smart mobile user to roam seamlessly between these technologies for best possible services. To ensure Always Best Connectivity, one of the main short comings to be addressed is efficient handoff management and there are a lot of works already published addressing various issues of efficient handoff management till date. This paper provides a comprehensive detail of different handoff management strategies reported so far in a systematic manner and also throws light on a particular scheme of handoff management between UMTS and WLAN providing QoS to the mobile users.
\end{abstract}

Index Terms-RSSI, SINR, VHO.

\section{INTRODUCTION}

A number of wireless communication technologies have been proposed and deployed in the last few years. Each of these technologies has advantages as well as disadvantages. All these technologies have been optimized for giving the smart mobile user the best possible data rate. But because of the complexity and environment specific nature, no single technology is able to provide the mobile users with sufficient data rate and good quality of service (QoS) in every situation. Therefore there is a strong need to compliment these technologies to meet user needs and provide them with Always Best Connectivity (ABC) [1] and service, thereby allowing them to be connected any time anywhere.

Different radio access technologies like GSM, WLAN, Universal Mobile Telecommunication System (UMTS), WiMax, LTE etc. have been incorporated in heterogeneous wireless technologies. WLAN is becoming very popular because of its low deployment cost and high communication rates (upto $54 \mathrm{Mbps}$ ) and are deployed as popular hot spots

where users can enjoy increased bandwidth within a limited coverage area. Similarly UMTS provides $2 \mathrm{Mbps}$ data rate within a large geographical area. Integration of these technologies drew attention of the researchers around the

Manuscript received November 15, 2014; revised June 5, 2015. This work is supported by the Department of Information Technology, Ministry of Communication and Information Technology, Govt of India (R\&D research project DeitY/IPR/1.2/28/2011 Dated 23.11.2012) for 2013-2014.

The authors are with the Assam Engineering College, Guwahati, Assam, India (e-mail: maushu@gmail.com, pathakdiganta1@gmail.com, iroykarmakar747@gmail.com). globe where an umbrella like topology can be set up with multiple WLANs operating under one UMTS. However several issues regarding heterogeneity to be addressed are mobility management, security, high QoS etc. Among them, mobility management is of great importance with respect to seamless roaming of users from one access network to another. Efficient Handover management ensures uninterrupted communication (data) during the time the MT (Mobile Terminal) changes its point of attachment. Traditionally handover process between same radio access technologies (Horizontal Handover) has been far simpler to implement than handover between diversified overlapping networks(Vertical Handover) where a MT needs to switch between different radio access technologies. A number of issues like user preference, QoS, Cost etc. are involved in switching from one type of radio access network to another.

In heterogeneous networks a MT can move across different access networks with different characteristics (coverage, bandwidth provided etc.) that offers different services to the users. During the handover process all these issues has to be taken into account to select the best network to which the MT has to be handed over.

Therefore, while performing the Vertical Handover (VHO) process the following important issues need to be resolved.

- How the (VHO) process works?

- Who will take the VHO decision, network or MT?

- Which VHO algorithm is used?

- Selection of the VHO criteria to be used like RSSI(Received Signal Strength Indicator), SINR etc or add other QoS parameters?

- How the information regarding various VHO criterion are collected?

In this paper, a detailed survey about VHO management and its issues are being written about. One of the many challenges to be addressed is automatic selection of the best possible network that enables seamless roaming in heterogeneous environment ensuring $\mathrm{ABC}$ [1]. The rest of the paper is organized as follows. In Section II, different aspects of VHO management in heterogeneous network has been discussed. Different interworking architecture between UMTS and WLAN are discussed in Section III. Section IV talks about different VHO decision strategies with special emphasis on a particular QoS based handoff scheme between UMTS and WLAN. A brief discussion about recent handover management in vehicular communication networks is also included in Section V. Finally Section VI concluded the paper.

\section{HANDOVER MANAGEMENT IN HETEROGENEOUS NETWORKS}

VHO management is the basic operation for any network 
that enables a MT to enjoy uninterrupted service during its movement from one point of attachment to another. VHO is performed because of the current access network is no longer able to service the MT or to distribute the load evenly among different mobile networks.

\section{A. VHO Process}

The implementation of VHO management are shown in Fig. 1 with three basic steps. Handover management process can be divided into three stages: initiation, decision and execution.

- Handover Initiation: Also known as System Discovery, information required to identify the need for handover are collected. As in this phase, the information of all neighbor networks are collected it can also be called system discovery phase.

- Handover Decision: This process finds the appropriate candidate network to which the MT can be handed over based on certain criteria (using some decision algorithm).

- Handover Execution: Finally signaling exchange for establishment of the new communication path has been performed along with data re-routing through this path.

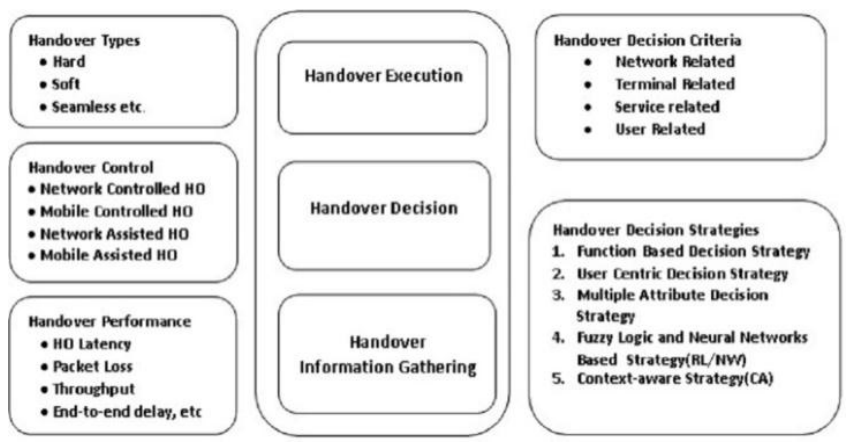

Fig. 1. Handover management concept [2].

\section{B. Types of Handover}

While implementing the handover procedure the main issue to be addressed is to preserve the quality of the communication link. The success of handover depends mainly upon how the new link has been established after the old ones are released. In this context two types of handover exist.

- Soft Handover: In this type of handover the MT keeps at least one connection with any of the access network during the entire handover period and it is referred to as make before break approach. This type of handover used in CDMA systems where MTs can communicate using different codes at the same time and same frequency [3].

- Hard handover: It is a break before make approach where the MT can keep only a single connection to a point of attachment at a time. This type of handover is found in TDMA and FDMA systems [3].

Apart from different handover types there are four methods which trigger a VHO

- Network Controlled Handover (NCHO): In this type of handover the network has the primary control in the entire process and takes handover decision so that the computational overhead at the central point of the system increases which may be the bottleneck of the system. The network may not be able to take the right decision due to the lack of knowledge about the current condition of the MT.
- Mobile Controlled Handover (MCHO): In this type handover the MT gathers information required for the handover process and make handover decision on its own.

- Network Assisted Handover (NAHO): In NAHO, MT uses the information collected by the network to make handover decision.

- Mobile Assisted Handover (MAHO): Here a MT assists the network by providing its current status information to help to take VHO decision.

Apart from all those classifications, more generally $\mathrm{VHO}$ can be classified as [4].

- Forced handoffs, triggered by physical events regarding network interfaces availability;

- User handoffs, triggered by user policies and preferences.

\section{Handover Decision Criteria}

In the handover initiation or system discovery phase, the system periodically checks to find out the candidate network to which the MT can be handed over. The discovery phase searches the available network to keep the QoS level above a certain threshold. In that case handover is initiated when the QoS is decreasing below a certain threshold. Different criterion considered for handover decision can be classified broadly into four categories [5] (see Fig. 2).

\section{1) Network related criteria}

- Coverage: RSSI is related to the network coverage areas and it tells whether a certain network is available to the MT or not.

- Bandwidth: It is another important network related criteria which has a direct effect on QoS.

- Load: In VHO process network load plays a vital role. Since the bandwidth is shared among the users in case of WLAN, higher the number of users lowers the allocated bandwidth per user is. Thus the prior knowledge of network load may prevent acceptance of new connections once the load is high.

- Link Quality: A number of metric can be considered as link quality indicators. These include:

1) Bit Error Rate (BER): BER is an important link quality measurement that provides information whether a specified application is supported by a network or not.

2) Signal-to-Interference Ratio (SINR): SINR qprovides an important measure of link quality which informs about performance degradation.

- Security: In some cases, confidentiality or integrity of the transmitted data may be important. For such type of situation, a network with higher security level may be chosen over another one that provides lower level data security. ${ }^{1}$

\section{2) Terminal related criteria}

- Velocity: The velocity of the MT is regarded as one of the most important handoff decision criteria. Fast moving MT may move out of range quickly. Therefore by handing over a fast moving mobile station from UMTS to WLAN can repeat the process back and forth.

- Battery Power: Power consumption has also a crucial issue. When the battery power is low then it is better to handover to a network that consumes less energy to extend the battery.

${ }^{1}$ Note: The word VHO and handover is used interchangeably throughout the paper having the same meaning. 


\section{3) User related criteria}

User preferences have effect on vertical handover decision in terms of QoS and monetary cost.

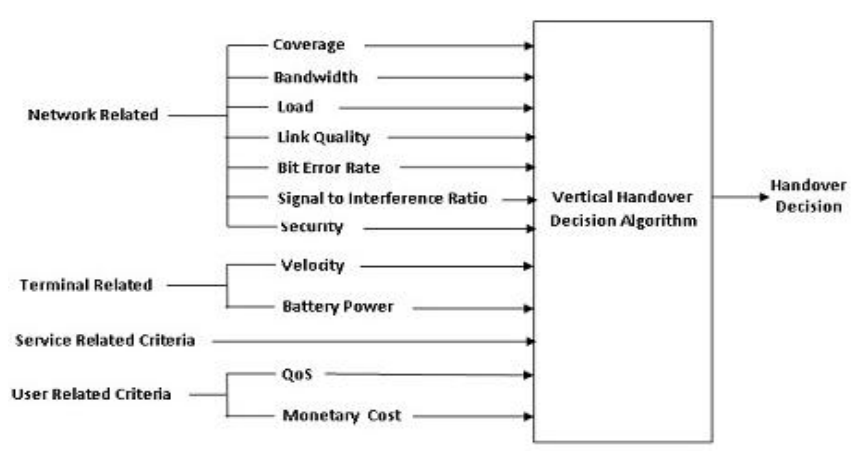

Fig. 2. Vertical handover decision criteria.

- QoS: User may prefer a certain level of QoS according to their ongoing application.

- Monetary Cost: Different network providers apply different billing schemes. This directly affects user preferences in turn effect the handover decision.

\section{4) Service related criteria}

Each individual network supports different types of service which have different data transfer rates, reliability level and suffers from different latencies. Therefore these criterions are regarded as important criteria for $\mathrm{VHO}$ decision.

\section{INTERWORKING BETWEEN HETEROGENEOUS NETWORKS}

Now a day's various wireless access technologies have been deployed and providing their services in the scenario. There is a strong need to integrate all those technologies in an optimal manner so that users can be always best connected [1]. The integration of these technologies is becoming a challenging task which draws attention to the researcher as well as the people working in telecommunication industry. Heterogeneous networks includes WLAN, Wireless Wide area network (WWAN), UMTS etc. WLAN provide higher data rate up to $200 \mathrm{Mbps}$ within a smaller coverage area. On the other hand UMTS provide data rate up to 2 Mbps within a greater coverage area. These two technologies are regarded as the best candidates for integration which could provide seamless services to the users. Several methods have been proposed in the literature to classify different internetworking architecture.

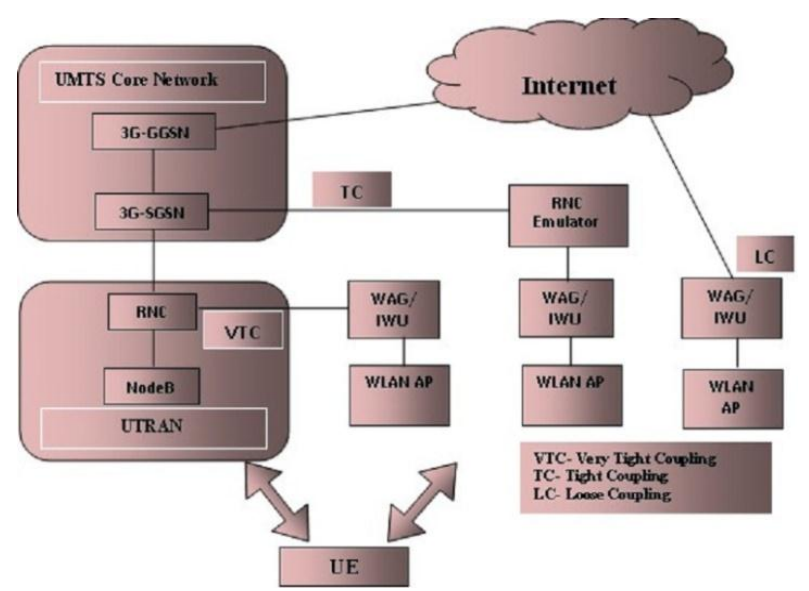

Fig. 3. Interworking approach.
The first standardization effort towards the categorization of different interworking has been made by ETSI [3]. To implement the UMTS-WLAN internetworking strategies three approaches are proposed in [6], [7] are Mobile IP Approach, Gateway Approach and Emulator Approach.

According to the categorization scheme followed in the article [3] different interworking architectures between 802.11 based WLANs and GPRS/UMTS networks fall into three major categories: loose, tight, and very tight coupling. All these interworking approaches are shown in Fig. 3. In Loose coupling approach, the two networks interconnected independently. They may share common authentication, authorization and accounting (AAA) information, but data flow from one network is independent of the other. This approach has several advantages. It allows independent deployment and traffic engineering of WLAN and UMTS can be easily adapted to the existing communication system. In tight coupling approach WLAN Access Points works as a NodeB and the WLAN traffic is injected to the UMTS core network. Although in tight coupling approach the handover latency is lower, the SGSN may be exhausted early with packets from both UMTS and WLAN. Another variation of tight coupling suggest in [3] is very tight coupling. In this approach WLAN Access Point is interworked at the UTRAN level with each WLAN AP working as NodeB.

\section{Vertical Handover DeCision STRATEgIeS}

Firstly one needs to be able to decide the best possible strategy to switch to the best network. A few such VHO algorithms are discussed here.

\section{A. Function Based Strategy}

An utility function (UF) which may be a cost function (minimum cost), an objective function with respect to certain constraint etc. can be used to drive the handover decision strategy. The VHO cost function is measured for all networks that cover the service area of a user. In policy enabled handover strategy described in [8] uses several parameters to calculate the cost function. The cost of using a network $\mathrm{n}$ depends on the bandwidth $\left(B_{n}\right)$ it can offer, the power consumption of using the network access device $\left(P_{n}\right)$, and the $\operatorname{cost}\left(C_{n}\right)$ of this network.

$$
\operatorname{Cost}_{n}=f\left(B_{n}, P_{n}, C_{n}\right)
$$

The cost function can be expressed as a sum of some normalized form of each parameter. Normalization is used to ensure that the sum of the values in different units is meaningful. The importance of each parameter may be specified by the user in terms of weight. The cost function of using a network $n$, named $f_{n}$, can be defined with $N(t)$ as the normalization function of parameter $t$ as:

$$
f_{n}=w_{b} \cdot N\left(1 / B_{n}\right)+w_{p} \cdot N\left(P_{n}\right)+w_{c} \cdot N\left(C_{n}\right)
$$

The cost function is periodically recalculated with updated parameters. The network with consistently better result will be selected as the target network. To get rid of the handover fluctuation popularly known as ping pong effect, the policy enabled handover decision uses a stability period. Stability 
period is the waiting time before handoff which is determined whenever the MT finds the target network.

The operating environment of policy-enabled handover is a Mobile IP-like infrastructure. Handover decision and operations are all done at the MT. All packets sent by the correspondent host to the MT passes through the home agent. The home agent routes the packet either to the multicast care of address or to unicast care-of-address of the MT. When the MT is in WLAN, the packets are first routed to the home agent first then to the correspondent node. To achieve seamlessness, the handoff latency must be low enough to ensure the continuity of the running applications. The system encourages user involvement (for policy specification) with minimum user interaction (implies automaton). For improving the system stability, it uses a performance agent that collects the information about current bandwidth status and periodically announces this information to its coverage area. Since all data goes through the base stations, they have the most accurate information on current bandwidth usage and the available bandwidth in the network. Based on this information, they design policy for load balancing avoiding handover synchronization using randomized stability period.

Chen et al. [9] proposed an adaptive scheme for vertical handoff in wireless overlay network by discovering all the reachable networks using the information about the MT and Location Service Server. This process can balance the power consumption and system discovery time. The authors use UF to evaluate the reachable networks. The UF quantifies the QoS provided by the wireless network from the viewpoint of the application running in the MT. When the MT finds a network which has higher UF than the current one, then the MT starts to observe whether it is consistently better than the current one. The time the MT observes the better network is called stability period [8]. The author introduces two adaptive handover decision methods adjusting the stability period, according to the network resources and the running applications on the MT.

\section{B. User Centric Decision Strategy}

User preferences in terms of cost and QoS are the most important and interesting policy parameter for VHO decision. In [10] the authors mainly focus their work to find an intelligent solution to radio access network (RAN) selection decision problem for non-real time data services (FTP). Selection of a network is based on the Consumer Surplus Value, which is the difference between the monetary value of the data to the user and the actual price charged. Different decision metrics has been proposed to find the appropriate UF. They are: risk neutral (user equally prefers paying less to experiencing less delay), risk seeking (user prefers alternative of less delay to assured money saving) and risk adverse (user prefers to be certain of paying less). The scenario considered here contains two partly overlapping WLANs, each with a number of overlapping traffic generating background traffic. The simulation model under consideration has a topology of wired network connected to the WLAN APs. The terminal connected to the WLAN APs is multi homed with inbuilt CS based network selection based strategy. The result reported here indicates that the behavior of the utility function dependent in the file size. Thus the value of employing an appropriate UF is highly dependent on the file size.
In [11] the author presents a user centric network selection strategy for non-real time traffic. MTs will survey the radio interface and determine a list of current available network at the time of access network selection decision. To predict the current transfer rate, an algorithm is employed in each of the selected radio access network. The author uses a basic rate prediction scheme, which takes the average of the last five data transfers on each of the different available network and uses this as the predicted rate for the next data transfer. The utility function taken here depends on the priority of the data, the delay tolerance levels etc.

\section{Multiple Attribute Decision Strategies (MAD)}

In next generation network, the VHO decision problem deals with making a choice among a number of candidate networks from different service providers based on different criteria. This is called a MADM(Multiple Attribute Decision Making) problem. In [12] Chai et al. mentioned a number of MADM based algorithms.

- Simple Additive Weighting(SAW): Here the overall score of a candidate network is determined by the weighted sum of all the attribute values [13], [14].

- Technique for Order Preference by Similarity to Ideal Solution (TOPSIS): Here the selected network is the closest to the ideal solution and farthest from the worst case solution [14]. The ideal solution is obtained by the best value of each metric. If $c_{i}{ }^{*}$ is the relative closeness (similarity) of the candidate network i to the ideal solution. The selected network $A_{T O P}$ is:

$$
A_{T O P}^{*}=\arg \max _{i \in M} C_{i}^{*}
$$

- Analytic Hierarchy Process (AHP): It decomposes the network selection problem into sub problems and assigns a weight for each problem [14], [15].

- Grey Relational Analysis (GRA): GRA makes a grey relationship and ranks the candidate networks. Finally it selects the one with highest ranking [14].

- Multiplicative Exponent Weighting (MEW): This is a MADM scoring method. Here in this approach the VHO problem can be expressed as a matrix form where each row $i$ corresponds to the candidate network i and each column $j$ corresponds to an attribute. The score $S_{i}$ of network $i$ can be expressed as the weighted product of the attributes [14].

$$
S_{i}=\prod_{j=1}^{N} x_{i j}{ }^{w_{j}}
$$

A comparison among SAW, TOPSIS, GRA and MEW is presented in [14] where the attributes considered for handoff decision are bandwidth, delay, jitter and BER. It shows that MEW, SAW and TOPSIS provide similar performance to the traffic classes used while GRA provides a slightly higher bandwidth and lower delay for interactive and background traffic classes.

\section{Fuzzy Logic and Neural Networks Based Strategies (FL/NN)}

Fuzzy Logic and Neural Networks are being used in decision making for the last few years. They can be used to choose the appropriate time and appropriate access network to handover among different available access network.

In [16] authors propose a solution using Fuzzy Logic 
where Mobile Satellite Networks are used to provide complementary service coverage to the terrestrial (GPRS and UMTS) counterparts. The handover decision algorithm proposed here selects a segment or network for a particular service which can satisfy certain objectives like low cost, good signal strength, optimum bandwidth, low network latency, high link availability, long battery life etc. for the preferred segment. The fuzzification procedure involves the evaluation and comparison of the available segments. The inputs received from the network provider and the user is used to evaluate the importance of each criteria. The segment selection procedure consists of two stages:

- In the first stage the data from the system (different criteria) are fed into the fuzzifier, which converts it into a fuzzy set. A fuzzy set has a clearly defined boundary and a varying degree of membership. In a fuzzy set, the cost of a segment can be represented by anything between 0 and 1 depending on the membership function. In order to obtain the representative value known as membership value, the measurements for a particular parameter are mapped onto a membership function. The weighting of different criteria actually evaluates the importance of different criteria. Analytic Hierarchy Process (AHP) is used to weighting a variety of criteria. Here in this process the segment selection criteria are identified first and then they are compared pair wise. Finally the weighting of different criterions are obtained.

- In the second stage, the weighting is applied to each of the criterions. As a result a decision function is obtained which satisfy all the decision objectives. The chosen segment is the segment with the maximum or highest membership values.

The first stage can be done before the handover initiation. The second stage is described in [17]. In [18] Horrich et al. proposed a fuzzy multi criteria VHO algorithm. The algorithm is based on fuzzy logic control(FLC) which taken into account multiple relevant criteria like the received energy per chip divided by the power density in the band (CPICH $\left.E_{c} / \mathrm{NO}\right)$ for UMTS and RSS for WLAN, load information and mobile terminal velocity. The fuzzy logic control (FLC) considered here is made up of a fuzzifier, an inference system and a defuzzifier. The inference system applies certain rules to the fuzzified values. The FLC based solution is further enhanced by Multi-Layer Back-Propagation neural network with adaptive Learning Rate and momentum which learns the relationship between the FLC parameters and adapts them to the traffic variation and the environment fluctuation.

\section{E. Context Aware Strategies}

This type of approach the handover decision is taken based on the context information of the MT and the available networks. From the context information collected from the MT and the network, MT or network finds out whether a handover is necessary and if necessary then the target network.

In [19] the author proposes an algorithm for VHO decision on the basis of context information collected from the current network as well as available networks. It consists of two main stages: Context Repository and Adaptability Manager. The context repository gathers, manages and evaluates context information and the Adaptability Manager makes decisions about context changes. The proxies residing in each network is responsible for handover execution. It follows a rule based handover and the rules decide whether a handover is necessary or not and which would be the alternate network. The network selection is based on QoS level of a network and the process is invoked when the QoS level is below the perceived acceptance quality. It has to satisfy multiple objectives including satisfying user's device preferences, achieving the highest level of bandwidth for applications while minimizing packet loss, delay, jitter, and avoiding bandwidth fluctuations which may affect the applications. The proposed solution gives a smart way of handover decision process based on the gathered context information.

In [20] the author Ahmed et al. describes a context-aware decision algorithm based on the AHP method. The algorithm takes into account context information from both the MT and network side. Here the services offered by a MT are divided into three service types as conversational/real-time services, interactive services and streaming services. For any of these three service types, a user needs to define three sets of relative priorities which are (i) objective priorities (ii) interface priorities and (iii) application priorities. User defined service preferences are categorized and compared with the capabilities of the available networks by employing the decision algorithm. The decision algorithm uses only basic mathematical calculations due to which the processing time is greatly reduced as well as it becomes suitable for embedded hardware in practical mobile devices. This algorithm minimizes processing time, handover delay, and CPU and memory usage because the whole process is executed only once for each type of running application.

A context-aware handover architecture is proposed based on active networking technology in [21]. The paper contributes an integrated architecture for context-aware handover and evaluation. The architecture consists of a programmable platform installed on network and mobile nodes and a service deployment framework capable of deploying different modules. Here context information is stored in context information repositories, such as the Location Information Server (LIS), Network Traffic Monitor (NTM) and the user's profile repository. Moreover, a Handover Manager (HM) is used which controls handovers carried out in some part of an access network and acts as a context collection point. A Service Deployment Server (SDS) is used to manage and install the service modules needed on the network nodes and mobile nodes. A flexible programmable platform is used to install different algorithms for different cases without interrupting the proper working of the node. With the aid of context exchange protocol up-to date context information is exchanged when needed for handover decision. The context management framework is in charge of collecting the relevant context information for different services and managing the context information. The programmable platform is used to download and install the suitable modules for context exchange.

\section{F. QoS Based Handover Scheme between UMTS and WLAN}

Finally, a QoS based handover mechanism over the ISN (Intermediate Switching Network) framework [22] is introduced here. This framework is based on a loose coupling 
architecture between UMTS and WLAN where connecting to faster WLAN is being preferred over UMTS network. This scheme uses SINR along with other QoS values like bandwidth etc. as handover criterion. An umbrella like topology is considered where IEEE 802.11 networks create coverage holes under UMTS coverage area. Whenever a MT enters under the coverage of IEEE 802.11 WLAN network, it is preferable to handover the connection from UMTS network to IEEE 802.11 WLAN network. The handover decision is taken cooperatively by the MT and its current point of attachment based on QoS requirement. As the UMTS network is available all the time, so whenever a MT is attached with IEEE 802.11 WLAN, it is always preferable to handover to another IEEE 802.11 WLAN, if available, compared to handover to the UMTS network. This is because, sudden performance degradation can be observed if the MT is handed over to the UMTS network from IEEE 802.11 WLAN network. Further, existing connections are preferred over new connections. This algorithm considers a per-node QoS requirement where the QoS for every mobile user is based on the economic subscription with the service provider. The scheme proposed here has following contributions.

- A per-node QoS differentiation strategy is proposed where mobile users can avail network service based on their economic subscription with the service provider.

- The new connections are admitted to the network if sufficient bandwidth is available after allocating required bandwidth to all the existing connections first.

- To maintain consistency in QoS, existing connections are preferred over new connections.

- A pre-handover bandwidth reservation scheme is proposed to reserve bandwidth at alternate connection points to avoid QoS degradation during handover

Further, pre-handover bandwidth reservation helps to prioritize the handover from one IEEE 802.11 network to another IEEE 802.11 network over the handover from UMTS to IEEE 802.11 network. A per-node QoS requirement is considered in terms of bandwidth, delay and jitter. The handover decision is based on SINR value along with the QoS requirements. The QoS parameters are prioritized based on their corresponding weight. For this purpose, a QoS parameter is defined called Normalized QoS Value (NQV) which computes the QoS requirement for a MT.

\section{Mobility Management In HeterogeneOUS NETWORKS}

The emerging trends of different applications for vehicular communications influence the researchers in conducting research in vehicular communication network especially in handover management. Vehicular ad-hoc network (VANET) includes vehicle-to-vehicle (V2V) and vehicle-to-infrastructure (V2I) network. V2I network refers to the communication between vehicles and infrastructure of roadside unit (RSU), e.g. base station and access point (AP) connected with Internet and $\mathrm{V} 2 \mathrm{~V}$ refers to the communication between vehicles in the VANET. To support intelligent transportation system (ITS) application Network Mobility Basic Support (NEMO BS) is used.

In [23] the author discusses about different challenges and solution of IP mobility management for vehicular networks. For efficient mobility management in vehicular communication network a special IP mobility mechanism is required while changing the point of attachment. The stack of protocols for vehicular network includes NEMO BS which enables IP mobility for infotainment applications. NEMO BS uses tunnel from Mobile Router home agent (MR-HA) to the Mobile network node (MNN) every time an MNN communicates with any correspondent node $(\mathrm{CN})$. This may effect the performance of some delay sensitive applications.

Many works has been done for host mobility in heterogeneous environment. There are a few works reported on network mobility (NEMO). In [24] the author reports a solution which consists of mobile DHCPv6 agents and handover management centre (HMC). Forward loss recovery and location management were implemented based on mobility prediction. Cooperative mobile router-based handover (CoMoRoHo) was also reported in [24]. It uses multihoming technique to reduce packet loss and handoff latency. As the highly mobile vehicles causes high handover rate, simultaneous mobility will occur frequently in vehicular networks. A simultaneous mobility solution for situation with network mobility has been reported in [25]. A proxy-aided simultaneous handover (PASH) mechanism for mobile networks in vehicular environment was proposed. This mechanism solves addressing problem of simultaneous handover.

\section{CONCLUSION}

In this paper, an overview of VHO process along with a comprehensive survey of different handover strategies documented so far has been presented. With the advancement in technology and the increasing user-centric service requirement from the MT. There is an increase in demand of infotainment applications resulting in scarcity of network resources which in turn increases the complexity of handover management.

It is well observed that advanced decision function and a combination of adding more QoS criterion is required for efficient handover decision achieving user satisfaction and making efficient use of the network resources.

\section{REFERENCES}

[1] E. Gustafsson and A. Jonsson, "Always best connected," IEEE Wireless Communications, vol. 10, issue 1, pp. 49-55, February 2003.

[2] M. Kassar, B. Kervella, and G. Pujolle, "An overview of vertical handover decision strategies in heterogeneous wireless networks," Computer Communications, vol. 31, no. 10, pp. 2607-2620, 2008.

[3] G. Lampropoulos, N. I. Passas, L. F. Merakos, and A. Kaloxylos, "Handover management architectures in integrated WLAN/Cellular networks," IEEE Communications Surveys and Tutorials, vol. 7, no. 4, pp. 30-44, 2005.

[4] M. Bernaschi, F. Cacace, A. Pescape, and S. Za, "Analysis and experimentation over heterogeneous wireless networks," in Proc. the First International Conference on Testbeds and Research Infrastructures for the Development of Networks and Communities (TRIDENTCOM05), pp. 182-191.

[5] M. Zekri, B. Jouaber, and D. Zeghlache, "A review on mobility management and vertical handover solutions over heterogeneous wireless networks," Computer Communications, vol. 35, pp 2055-2068, 2012.

[6] S. L. Tsao and C. C. Lin, "Design and evaluation of UMTS-WLAN interworking strategies," in Proc. the Vehicular Technology Conference, 2002, pp. 777-781.

[7] T. Soungalo, L. Renfa, Z. Fanzi, \& H. N. Waita, "Performance analysis of interworking between WLAN and 3G networks based on three approaches," Procedia Engineering, vol. 29, pp. 1126-1132, 2012. 
[8] H. J. Wang, R. H. Katz, and J. Giese, "Policy-enabled handoffs across heterogeneous wireless networks," in Proc. Second IEEE Workshop on Mobile Computing Systems and Applications, 1999, pp. 51-60.

[9] W. Chen, J. Liu, and H. Huang, "An adaptive scheme for vertical handoff in wireless overlay networks," in Proc. the 10th International Conference on Parallel and Distributed Systems, 2004, pp. 541-548.

[10] O. Ormond, J. Murphy, and G. Muntean, "Utility-based intelligent network selection in beyond $3 \mathrm{G}$ systems," in Proc. IEEE International Conference on Communications (ICC 2006), 2006, vol. 4, pp. 1831-1836.

[11] O. Ormond, P. Perry, and J. Murphy, "Network selection decision in wireless heterogeneous networks", in Proc. IEEE 16th International Symposium on Personal Indoor and Mobile Radio, Communications, September 2005, vol. 4, pp. 2680-2684.

[12] R. Chai, W.-G. Zhou, Q.-B. Chen, and L. Tang, "A survey on vertical handoff decision for heterogeneous wireless networks," in Proc. IEEE Youth Conference on Information, Computing and Telecommunication, September 2009, pp. 279-282.

[13] S. M. Liu, S. Pan, Z. K. Mi, Q. M. Meng, and M. H. Xu, "A simple additive weighting vertical handoff algorithm based on SINR and AHP for heterogeneous wireless networks," in Proc. International Conference on Intelligent Computation Technology and Automation, May 2010, vol. 1, pp. 347-350.

[14] E. Stevens-Navarro and V. W. Wong, "Comparison between vertical handoff decision algorithms for heterogeneous wireless networks," in Proc. IEEE 63rd Vehicular Technology Conference, May 2006, vol. 2, pp. 947-951.

[15] Q. Song and A. Jamalipour, "A network selection mechanism for next generation networks," in Proc. IEEE International Conference on Communications, May 2005, vol. 2, pp. 1418-1422.

[16] P. Chan, Y. Hu, and R. Sheriff, "Implementation of fuzzy multiple objective decision making algorithm in a heterogeneous mobile environment," in Proc. IEEE Wireless Communications and Networking Conference, 2002, vol. 1, pp. 332-336.

[17] P. Chan, Y. Hu, and R. Sheriff, "Implementation of fuzzy multiple objective decision making algorithm in a heterogeneous mobile environment," in Proc. IEEE Wireless Communications and Networking Conference, 2002, vol. 1, pp. 332-336.

[18] S. Horrich, S. B. Jamaa, and P. Godlewski, "Adaptive vertical mobility decision in heterogeneous networks," in Proc. International Conference on Wireless and Mobile Communications, 2007, p. 44.

[19] S. Balasubramaniam and J. Indulska, "Vertical handover supporting pervasive computing in future wireless networks," Computer Communications, vol. 27, issue 8, May 2004, pp. 708-719.

[20] T. Ahmed, K. Kyamakya, and M. Ludwig, "A context-aware vertical handover decision algorithm for multimode mobile terminals and its performance," in Proc. the IEEE/ACM Euro American Conference on Telematics and Information Systems (EATIS 2006), 2006, pp. 19-28.

[21] Q. Wei, K. Farkas, C. Prehofer, P. Mendes, and B. Plattner, "Context aware handover using active network technology," Computer Networks, vol. 50, issue 15, 2006, pp. 2855-2872.

[22] M. Barooah, S. Chakraborty, S. Nandi, and D. U. Kotwal, "An architectural frame-work for seamless handoff between IEEE 802.11 and UMTS networks," ACM/Springer Journal of Wireless Networks, vol. 19, issue 4, pp. 411-429, May 2013.

[23] S. Cespedes, X. Shen, and C. Lazo, "IP mobility management for vehicular communication networks: Challenges and solutions," IEEE Communications Magazine, vol. 49, issue 5, pp. 187-194, May 2011.

[24] K. Zhu, D. Niyato, P. Wang, E. Hossain, and D. Kim, "Mobility and handoff management in vehicular networks: A survey," Wireless Communications and Mobile Computing, vol. 11, issue 4, pp. 459-476.

[25] W. K. Chiang, W. Y. Chang, and L. Y. Liu, "Simultaneous handover support for mobile networks on vehicles," in Proc. IEEE WCNC, 2008, pp. 2771-2776.

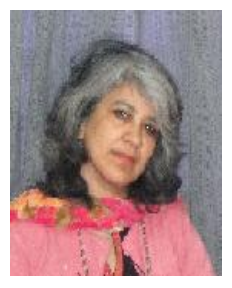

Maushumi Barooah graduated in mathematics from Guwahati University and then did her master program in computer applications and received her master degree in computer science and engineering from Indian Institute of Technology (IIT) Guwahati, Assam, India. She has completed her Ph.D. degree in computer science \& engineering from IIT Guwahati. She is currently working as an associate professor at Assam Engineering College, Guwahati, Assam, India. She is presently working in handoff management in heterogeneous networks. Her research interests are wireless networks and heterogeneous networks.

Diganta Kumar Pathak graduated in physics and then did his master's program in computer applications followed by a MTech degree in information technology from Gauhati University, Assam, India, in 2007, 2010 and 2013 respectively. Presently he is working as a junior research fellow in the Department of Computer Applications, Assam Engineering College. His area of interest includes wireless networks and heterogeneous networks.

Ishita Roy Karmakar completed her degree in engineering in the field of computer science from Jorhat Engineering College in the year of 2012. Currently she is working as a junior research fellow in the Department of Computer Applications, Assam Engineering College. 